\title{
PLASMODIES DE LÉMURIENS MALGACHES ${ }^{1}$
}

\author{
I. LANDAU*, J.-P. LEPERS**, L. RABETAFIKA***, \\ D. BACCAM*, W. PETERS****, P. COULANGES**
}

RÉSUMÉ. L’étude des Plasmodium d'un Lémurien malgache, Lemur macaco macaco provenant de la région d'Ambanja, révèle un polyparasitisme par quatre espèces différentes :

- Plasmodium coulangesi décrit récemment par Lepers et coll. (1989).

- Plasmodium bucki n. sp. : ses principaux caractères différentiels sont le nombre élevé (32) de mérozoïtes des schizontes mûrs et la présence de taches de type "Maurer " dans un globule rouge hypertrophié et plus rosé que les cellules saines.

- Plasmodium percygarnhami n. sp. (= Plasmodium girardi sensu Uilenberg, 1970 pro parte et sensu Garnham et Uilenberg, 1975 pro parte) caractérisé par le nombre de mérozoïtes dans le schizonte mûr (20) et par les altérations de la cellule hôte (déformations en feuille de houx ou en oursin et décoloration chez les formes âgées).

- (?) Plasmodium lemuris; les gamétocytes sont de très grande taille $(11 \mu \mathrm{m} \times 7 \mu \mathrm{m})$ parasitant un globule rouge très hypertrophié $( \pm 10 \mu \mathrm{m})$, déformé et de coloration rosée. L’hypothèse selon laquelle il pourrait s'agir d'un Haemoproteidae est avancée.

L'analyse des données de la littérature amène les auteurs à modifier la nomenclature précédemment établie pour les espèces suivantes :

- Plasmodium girardi Buck et coll., 1952, sensu Garnham, 1966, sensu Uilenberg, 1970 pro parte et sensu Garnham et Uilenberg, 1975 pro parte, est désigné sous le nom de Plasmodium sp. pour les formes décrites chez Lemur fulvus fulvus; le taxon P. percygarnhami désigne les formes de Lemur m. macaco et P. girardi Buck et coll., 1952, les formes de Lemur fulvus rufus.

- Plasmodium foleyi Buck et coll., 1952, sensu Garnham et Uilenberg, 1975, parasite de L. f. fulvus est nommé Plasmodium uilenbergi n. sp., P. foleyi étant un parasite de L. f. rufus.

Le cas de P. lemuris étant mis à part, du fait qu'il n'appartient peut-être pas au genre Plasmodium, l'analyse morphologique nous conduit à individualiser 7 espèces, chez les trois Lémuriens actuellement étudiés. Il apparaît ainsi un phénomène de vicariance comparable à ce que l'on connaît chez les Rongeurs africains, mais avec une spéciation plus poussée.

1. Travail ayant bénéficié de l'appui du Programme Spécial/PNUD/Banque Mondiale/OMS de Recherche et de Formation concernant les Maladies Tropicales.

* Laboratoire de Protozoologie et Parasitologie comparée, EPHE et Laboratoire de Zoologie (Vers) associé au CNRS, Muséum National d'Histoire Naturelle, 61, rue Buffon, F 75231 Paris Cedex 05

** Institut Pasteur de Madagascar, Boite Postale 1274, Antananarivo, Madrgascar.

*** Laboratoire de Zoologie, Université de Madagascar, Antananarivo, Madagascar.

**** London School of Hygiene and Tropical Medicine, Keppel Street, Londres.

Accepté le 13 décembre 1988. 
Ces formes vicariantes constituent un couple formé par :

- d'une part une petite espèce, siégeant dans une hématie de taille normale : P. girardi chez $L$. $f$. rufus, P. sp. chez L. $f$. fulvus, P. percygarnhami, avec, en outre, P. coulangesi, chez L. $m$. macaco.

- d'autre part une grande espèce entraînant une hypertrophie du globule rouge : $P$. foleyi chez L. f. rufus, P. uilenbergi chez L. f. fulvus et P. bucki chez L. m. macaco.

Mots-clés : Plasmodium girardi, Plasmodium foleyi. Plasmodium lemuris. Plasmodium percygarnhami. Plasmodium coulangesi. Plasmodium uilenbergi. Lemur macaco macaco. Lemur fulvus fulvus. Lemur fulvus rufus. Vicariance. Madagascar.

\section{Plasmodia of Lemurs from Madagascar.}

SUMMARY. Lemur macaco macaco from Ambanja region was found polyparasitized by four different species of Plasmodium:

- Plasmodium coulangesi recently described by Lepers et al. (1989).

- P. bucki n. sp.: its main differencial characteristics are the large number (32) of merozoites produced in mature schizonts and the stippling, resembling Maurer's dots, in an hypertrophied pinkish erythrocyte.

- P. percygarnhami n. sp. (= P. girardi sensu Uilenberg, 1970 pro parte and sensu Garnham et Uilenberg, 1975 pro parte) producing 20 merozoites in mature schizonts and developing inside a deformed corpuscle (holly leaf-shaped or sometimes sea-urchin-shaped) which may also become decolourized when parasitized by older stages.

- (?) Plasmodium lemuris: gametocytes are very large $(11 \mu \mathrm{m} \times 7 \mu \mathrm{m})$; the parasitized erythrocyte is much hypertrophied $( \pm 10 \mu \mathrm{m})$, distorted and of a pinkish colour; one schizont only, possibly exo-erythrocytic, was found. The authors hypothesized this parasite to be a Haemoproteid.

The analysis of published data led the authors to make the following modifications to the nomenclature previously established:

- Plasmodium girardi Buck et al., 1952, sensu Garnham, 1966, sensu Uilenberg, 1970 pro parte and sensu Garnham and Uilenberg, 1975 pro parte, is refered to as Plasmodium sp., for stages developing in the blood of Lemur fulvus fulvus. The taxon P. percygarnhami is to be employed for stages developing in L. m. macaco and P. girardi Buck et al., 1952 for those developing in Lemur fulvus rufus.

- Plasmodium foleyi Buck et al., 1952, sensu Garnham and Uilenberg, 1975 in L. f. fulvus is named Plasmodium uilenbergi n. sp., P. folleyi being a parasite of L. $f$. rufus.

Excluding P. lemuris which probably does not belong to the genus Plasmodium, the morphological analysis led to individualize 7 species, in the three species of Lemurs studied. A phenomenon of "vicariance " thus appears, similar to what is known for the african Rodent Plasmodia, but with a more pronounced speciation.

The vicariant species form a pair constituted of:

- on the one hand a small species developing in a red blood cell of normal size, P. girardi in L. f. rufus, P. sp. in L. f. fulvus, P. percygarnhami, with also, P. coulangesi, in L. m. macaco; - on the other hand a large species determining an hypertrophy of the erythrocyte, $P$. foley $i$ in L. f. rufus, P. uilenbergi in L. f. fulvus and P. bucki in L. m. macaco.

Key-words: Plasmodium girardi. Plasmodium foleyi. Plasmodium lemuris. Plasmodium percygarnhami. Plasmodium coulangesi. Plasmodium uilenbergi. Lemur macaco macaco. Lemur fulvus fulvus. Lemur fulvus rufus. Vicariance. Madagascar. 
L'étude des Plasmodium de deux Lemur macaco macaco L., 1766, de la région d'Ambanja au nord de Madagascar et la transmission expérimentale à un autre Lémurien a permis d'individualiser 4 espèces distinctes : Plasmodium coulangesi Lepers et coll., 1988, Plasmodium bucki n. sp., Plasmodium percygarnhami n. sp. et Plasmodium lemuris Huff et Hoogstraal, 1963.

La morphologie de chaque espèce est caractéristique ; il devient donc possible, en analysant les données bibliographiques, de penser que chaque espèce ou sousespèce de Lémurien a ses propres Plasmodium.

La classification des Lémuriens utilisée est celle de Petter et coll. (1977).

\section{Matériel biologique}

\section{LÉMURIENS ÉTUdiÉS}

Les descriptions ont été faites à partir des frottis de sang de trois Lemur macaco macaco.

- Lémur 1 naturellement infecté est la femelle chez laquelle a été décrit $P$. coulangesi Lepers et coll., 1988. Il a été splénectomisé le 13.3 .1987 ; il avait alors une parasitémie de 0,01\%.

— Lémur 2 naturellement infecté est un mâle maintenu en captivité à l'Institut Pasteur de Madagascar depuis novembre 1985 ; faiblement infecté à son arrivée au Muséum (parasitémie de 0,02 \%) le 4.12.1987, essentiellement par de jeunes trophozoïtes; il est splénectomisé le 9.12.1987.

- Lémur 3 infecté expérimentalement est une femelle maintenue en captivité au parc Zoologique de Tsimbazaza depuis novembre 1983 ; arrivé au Muséum, comme le précédent, le 4.12.1988; deux frottis effectués, l'un le jour de son arrivée, l'autre 21 jours plus tard sont négatifs.

Le 4.1.1988 et le 6.1.1988 il est inoculé par voie intra-veineuse avec des sporozoïtes obtenus par dissection de glandes salivaires d'Anopheles stephensi gorgés sur le Lémur 2 (cf. Rabetafika et coll. 1989, sous presse).

\section{Allure générale des infections}

LÉMUR 1 : EÉvolution de la parasitémie : selon Lepers et coll., la parasitémie a atteint 14,5\%, J12 après la splénectomie ; un traitement par la quinine a fait chuter rapidement l'infection et deux recrudescences mineures ont été notées à J41 et J58. - Espèces : au cours du premier pic trois espèces sont présentes, $P$. coulangesi et $P$. percygarnhami abondants et en nombre à peu près équivalent et $P$. lemuris, très rare. Au cours des $2^{\mathrm{e}}$ et $3^{\mathrm{e}}$ pics, seul $P$. percygarnhamia a été décellé. 
LÉMUR 2 : - Évolution de la parasitémie : la parasitémie commence à augmenter aussitôt après la splénectomie pour atteindre $10,3 \%$ après 8 jours (J8). Un traitement de trois jours par la quinine est alors commencé. A J9 la parasitémie a encore augmenté; à J10, J11 et J12 la parasitémie diminue et les parasites présents sont en cours de destruction. Aucun frottis n'a été effectué du $13 \mathrm{e}$ au 19 e jours. A J20 le sang est apparemment négatif. La première recrudescence débute vers J25. La parasitémie augmente lentement pendant une semaine puis présente une augmentation brutale à J32 où elle passe en 24 heures de 0,8 à $3 \%$. Un deuxième traitement est décidé à J38 alors que la parasitémie est de $10 \%$. L’infection redevient inapparente. - Espèces : au cours du premier accès suivant la splénectomie $P$. coulangesi est nettement prédominant. $P$. percygarnhami est présent mais à un taux très faible. Les deux autres espèces, Plasmodium bucki et $P$. lemuris ne sont pas visibles. Après traitement par la quinine, au cours du deuxième accès, la proportion de $P$. percygarnhami a beaucoup augmenté. Les deux espèces $P$. coulangesi et $P$. percygarnhami sont abondantes. Plasmodium bucki et Plasmodium lemuris sont observés mais leur parasitémie reste très faible.

LÉMUR 3 :-Évolution de la parasitémie : les premiers parasites sanguins apparaissent le 13.1.1988 (J9 après l'inoculation de sporozoïtes). Le 21.1.1988 (J21) la parasitémie étant de $4 \%$, et l'animal semblant fatigué, un traitement par la quinine est commencé. Le sang paraît se négativer rapidement. Une parasitémie inférieure à $0,5 \%$ est décelée le 8.02.1988. Le Lémurien n'a plus été suivi depuis. - Espèces : au cours du premier accès, suivant l'inoculation de sporozoïtes, trois espèces sont décelées : l'espèce prédominante est Plasmodium percygarnhami, Plasmodium bucki est relativement peu abondant, Plasmodium coulangesi est très rare. Après traitement par la quinine, une petite recrudescence de l'infection survient; la parasitémie à Plasmodium coulangesi et à $P$. percygarnhami a apparemment disparu, seul Plasmodium bucki est présent.

\section{Description morphologique}

Description De Plasmodium percygarnhami n. SP. (Planche I, 9 à 13, p. 170)

Le matériel holotype est un schizonte marqué au diamant, sur un frottis de sang du Lémur 3, déposé au Muséum et enregistré sous le numéro PX 118.

Trophozoütes : les plus petits parasites observés ont une taille légèrement supérieure à celle du mérozoïte ; ils se présentent comme une masse rose irrégulière, dans laquelle, le cytoplasme n'est pas distinct du noyau.

Le stade suivant est encore très petit; le noyau de grande taille forme une calotte, le cytoplasme une petite masse bleue qui lui est accolée ; il n'y a encore, ni vacuole ni pigment. 
En grandissant, le jeune trophozoïte, arrondi, présente un contour paraissant irrégulier en raison de la présence de minuscules vacuoles périphériques. Une petite vacuole apparaît contre le noyau.

Jusqu'à ce stade, le globule rouge est normal ; notons que les infections doubles ne sont pas rares.

Lorsque le trophozoïte atteint environ le $1 / 5 \mathrm{du}$ volume de l'hématie, les déformations caractéristiques de cette espèce apparaissent. Elles permettent dans les infections multiples par plusieurs espèces de repérer immédiatement la présence de $P$. percygarnhami. Le contour du globule rouge perd sa forme arrondie et présente des pointes ou des angles plus ou moins aigus; sa forme évoque souvent une "feuille de houx ».

Le trophozoïte, lui-même est de forme générale arrondie, parfois ovalaire, à contour irrégulier. Le noyau contient souvent un grain de chromatine très dense. La vacuole est unique ou fragmentée en plusieurs petites vacuoles qui siègent à proximité du noyau. Le pigment apparaît, sous forme de fins granules.

Lorsque le trophozoïte atteint une taille d'environ $1 / 3$ de celle de l'hématie il est compact, avec un contour un peu irrégulier, pouvant paraître crénelé en raison de la présence de très petites vacuoles périphériques ou de fins prolongements cytoplasmiques. Le noyau est de grande taille (le tiers ou la moitié du volume du trophozoïte), en position latérale. La vacuole juxtanucléaire du jeune trophozoïte a disparu ; le pigment est constitué par une masse compacte noire ou par des granules grossiers rassemblés dans une vacuole, proche du bord du schizonte.

Schizontes : la première division du noyau aboutit à une forme binucléée avec deux grands noyaux proches l'un de l'autre.

Le schizonte jeune à 5 ou 6 noyaux est rond et bien limité ; les noyaux sont grands, relativement homogènes, périphériques, entourant une zone cytoplasmique centrale bleu vif. Le pigment, comme dans le trophozoïte âgé, est rassemblé dans une vacuole excentrée.

Lorsque le nombre de noyaux augmente, ils envahissent également le centre du parasite; ils sont alors difficiles à distinguer les uns des autres; on observe un fond rouge sur lequel se détachent des masses chromatiniennes plus denses, rouge foncé. Le pigment est le plus souvent latéral. Le nombre de noyaux des schizontes mûrs, ou ayant apparemment achevé leurs divisions peut varier de 16 à 20, mais dans la grande majorité des cas il est de 20.

Le globule rouge parasité est toujours visible. Les déformations, déjà nettes lorsqu'il contenait un trophozoïte sont encore plus importantes, contrastant avec la forme arrondie du schizonte. Il est fréquemment allongé, anguleux, parfois comparable à l'hématie parasitée par $P$. ovale avec une extrémité pointue, l'autre crénelée; enfin il peut également avoir une forme en oursin. Le globule rouge, généralement de coloration normale, peut être décoloré par les formes âgées. Enfin son volume reste inchangé ou légèrement augmenté.

Gamélocytes : les gamétocytes de $P$. percygarnhami sont denses et chromophiles, arrondis ou légèrement ovalaires, à contour régulier. Quelques fins grains 
azurophiles sont inconstamment présents. Ils mesurent 5 à $6 \mu \mathrm{m}$ et n'entraînent pas d'hypertrophie de leur cellule hôte.

Le globule rouge est parfois invisible mais le plus souvent il est en oursin, formant de petits prolongements pointus autour du parasite.

Le microgamétocyte a un grand noyau granuleux, parfois réticulé, arrondi, central, entouré par une bande de cytoplasme bleu mauve, foncée. Le granule chromatinien accessoire est présent mais se distingue mal dans un noyau rouge. Le pigment en granules inégaux, plutôt arrondis, noirs avec des reflets jaunâtres est réparti sur une ou deux petites zones, voisines. Il chevauche le noyau.

Le macrogamétocyte a des caractères communs avec le microgamétocyte : forte chromophilie, hématie hôte déformée, présence de quelques fins granules azurophiles. Son noyau est plus petit, il ne possède pas de granule chromatinien accessoire, et le pigment est plus rassemblé.

Description de Plasmodium bucki N. SP. (Planche I, 14 à 20, p. 170)

Le matériel holotype est un schizonte marqué au diamant, sur un frottis de sang du Lémur 3, déposé au Muséum et enregistré sous le numéro VII 223.

Trophozoütes : les anneaux n'ont pas été identifiés. Les trophozoïtes jeunes et âgés sont, par contre, facilement reconnaissables à leurs caractères morphologiques et à leur effet sur le globule rouge.

Leur forme est grossièrement arrondie ou ovalaire, leur contour est irrégulier avec de petits prolongements amiboïdes et de petites vacuoles périphériques; le noyau est relativement grand ( $1 / 4 \mathrm{du}$ parasite), le cytoplasme contient quelques petites vacuoles et forme des amas bleus entre les vacuoles. Le pigment à ce stade est représenté par quelques grains fins noirs et dispersés.

Le globule rouge est hypertrophié et de coloration plus rosée que les cellules voisines. La taille normale d'un globule rouge est de 5 à $6 \mu \mathrm{m}$; lorsqu'il est parasité, il peut atteindre $9 \mu \mathrm{m}$, mais le plus souvent sa taille se situe autour de $7 \mu \mathrm{m}$. Il peut être déformé, avec des prolongements mousses, ou un bord légèrement crenelé, mais n'a jamais la forme en "feuille de houx » ou en " oursin » observée chez $P$. percygarnhami. Un organite rouge, rarement plus d'un, de forme variable, rappelant les taches de Maurer est, le plus souvent, accolé au parasite.

Les infections doubles ne sont pas rares.

Schizontes : le jeune schizonte à 2 noyaux est déjà de grande taille $(6 \mu \mathrm{m})$. Il siège dans une hématie déformée, au contour irrégulier, mesurant 7 à $8 \mu \mathrm{m}$ de diamètre.

Avec 7 ou 8 noyaux, le schizonte remplit les $4 / 5$ de sa cellule ; il a une forme irrégulière; les noyaux sont relativement grands, granuleux, tantôt mal individualisés, tantôt bien détachés les uns des autres et formés par 4 grains de chromatine entourant un centre clair. Le cytoplasme est bleu assez soutenu contenant quelques petites vacuoles blanches; les grains de pigment sont individualisés, noir jaunâtres, plus grossiers, que précédemment et ils commencent à se rassembler sur une petite zone, le plus souvent latérale, parfois centrale. 
Au cours de son évolution le parasite occupe une place de plus en plus important dans le globule rouge. Lorsque les schizontes sont mûrs ou proches de la maturité le cytoplasme du globule rouge est réduit formant en périphérie, une bordure irrégulière ou des petites excroissances ; l'enveloppe de l'hématie est rosée, le cytoplasme résiduel est décoloré avec des tâches roses.

Trois schizontes mûrs, mesurant $8 \mu \mathrm{m}$ ont été observés ; deux d'entre eux contiennent 32 mérozoïtes et le troisième 34 .

Gamétocytes : nous n'avons observé que quelques macrogamétocytes et un seul microgamétocyte de cette espèce ; ils sont de forme arrondie et mesurent $7 \mathrm{um} \mathrm{de} \mathrm{diamètre;} \mathrm{ils} \mathrm{remplissent} \mathrm{presque} \mathrm{totalement} \mathrm{le} \mathrm{globule} \mathrm{rouge} \mathrm{encore}$ visible sous forme d'un liséré rose, hérissé de quelques prolongements triangulaires ou en forme de spicules. La tache satellite de type Maurer est allongée entre le parasite et le bord de l'hématie. Quelques rares granules azurophiles épars peuvent être notés dans les deux sexes.

Le macrogamétocyte a un noyau de grande taille (1/3 à $1 / 4$ du parasite), bien limité rose foncé, homogène, parsemé de quelques grains de chromatine plus denses; la couleur bleu vif de son cytoplasme est très caractéristique ; le pigment est noir à reflets jaunes en gros granules allongés disposés sur environ $1 / 5$ du cytoplasme et en quelques granules plus fins éparpillés.

Le seul microgamétocyte observé a un noyau granuleux, bien limité, occupant presque tout le parasite ; le cytoplasme forme une mince bande périphérique mauve. Le pigment est représenté par quatre gros granules noirs à reflets jaunes, chevauchant le noyau.

Compléments a LA Description de Plasmodium lemuris (Planche I, 21 à 24, p. 170)

Seuls quatre gamétocytes jeunes et mûrs ont été trouvés chez le Lémur 2. Les caractères les plus frappants sont la grande taille des parasites et les modifications de la cellule hôte.

Ils occupent un globule rouge très hypertrophié, de forme irrégulière, et dans un cas un prolongement lobaire a été observé.

Deux gamétocytes immatures ont été observés :

- un stade très jeune, grossièrement ovalaire, occupant le $1 / 3$ d'un globule rouge hypertrophié à $9 \mu \mathrm{m}$; le noyau occupe plus de la moitié du parasite; il est bien limité et homogène; le cytoplasme est bleu soutenu et le pigment en granules détachés, assez fins avec des reflets brun jaunâtres ;

- un jeune macrogamétocyte, occupant la moitié d'un globule rouge considérablement hypertrophié $(11 \mu \mathrm{m} \times 10 \mu \mathrm{m})$. Le cytoplasme est bleu vif, le noyau relativement petit et allongé, le pigment en grains bruns foncés avec des reflets jaunes, dispersés dans tout le cytoplasme.

Deux gamétocytes mûrs ont été vus :

— un microgamétocyte mesurant $11 \mu \mathrm{m} \times 6,5 \mu \mathrm{m}$, ovalaire, avec un contour 
net et régulier; le noyau est b:en limité occupant presque tout le parasite; il est entouré par une bande fine de cytoplasme bleu contenant quelques granules azurophiles. Le pigment est dispersé, fin, chevauchant le noyau;

- un macrogamétocyte, mesurant $11 \mu \mathrm{m} \times 7 \mu \mathrm{m}$; le noyau est bien limité et grossièrement rectangulaire ; le pigment est en grains arrondis ou ovalaires, bruns, dispersés. Le globule rouge déformé, mesure $13 \mu \mathrm{m} \times 8 \mu \mathrm{m}$.

\section{Discussion}

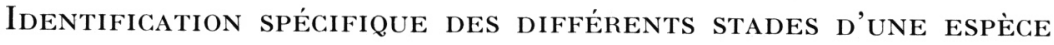

Lors des infections polyparasitaires il peut être difficile de déterminer à quelle espèce appartiennent les différents types de stades observés.

Tableau I. - Principaux caractères morphologiques des 7 plasmodies de Lémuriens connues.

\begin{tabular}{|c|c|c|c|c|c|c|c|}
\hline GLOBULE ROUGE & $\begin{array}{l}\text { Coulangesi } \\
\text { L.m.macaco }\end{array}$ & $\begin{array}{l}\text { girardi } \\
\text { L.t.rufus }\end{array}$ & $\begin{array}{c}\text { percygarnhami } \\
\text { L.m.mocaca }\end{array}$ & $\begin{array}{c}\text { sp } \\
\text { L.t.fulvus }\end{array}$ & $\begin{array}{l}\text { foleyi } \\
\text { L.f.rufus }\end{array}$ & $\begin{array}{c}\text { bucki } \\
\text { L.m.macaco }\end{array}$ & $\begin{array}{l}\text { uilenbergi } \\
\text { L.f.fulvus }\end{array}$ \\
\hline Hypertrophie & - & - & - & - & + & + & + \\
\hline Contour & Régulier & Régulier & $\begin{array}{l}\text { Feuille de houx, } \\
\text { oursin }\end{array}$ & & Régulier & Lobé & Régulier \\
\hline & Normale & Normale & $\begin{array}{l}\text { Décoloration } \\
\text { tardive }\end{array}$ & & Irès décoloré & Rosé & Normale \\
\hline Granulations & - & - & - & & - & type Maurer & type Ziemann \\
\hline IROPHOZOIIE & & & & & & & \\
\hline Vacuole & Peu visible & $\begin{array}{c}\text { Paraissant trover } \\
\text { le } B R\end{array}$ & Peuvisible & & Multiple & Petite & Peu visible \\
\hline Pigment & $\begin{array}{l}\text { Apparement } \\
\text { externe }\end{array}$ & Tardif & $\begin{array}{l}\text { Dans vacuole } \\
\text { périphér que }\end{array}$ & & $\begin{array}{c}\text { Amas } \\
\text { dissémines }\end{array}$ & Fin & Fin \\
\hline $\begin{array}{l}\text { Forme } \\
\text { SCHIZONIE }\end{array}$ & Arrondie & Arrondie & Arrondie & & Amíboïde & Arrondie & Arrondie \\
\hline Pigment & $\begin{array}{l}\text { Apparement } \\
\text { externe }\end{array}$ & Latéral & Latéral & Central & & Latéral & Peuvisible \\
\hline Nombre merozoites & 6 & $8-10$ & 20 & $12-16$ & & 32 & : \\
\hline GAMETOCYTES & & & & & & & 1 \\
\hline $\begin{array}{l}\text { Volume du GR } \\
\text { occupé }\end{array}$ & $9 / 10$ & $10 / 10$ & $9 / 10$ & $10 / 10$ & & $9 / 10$ & $3 / 4$ \\
\hline Taille & 6 à $6,5 \mu \mathrm{m}$ & 7 à $8 \mu \mathrm{m}(?)$ & 5 à 6 um & & & $7 \mu \mathrm{m}$ & $10-11 \mu \mathrm{m}$ \\
\hline Chromophilie & - & + & + & & & ++ & + \\
\hline Grains azurophiles & ++ & - & + & & & + & + \\
\hline $\begin{array}{l}\text { Gran. chromat. } \\
\text { accessoire }\end{array}$ & Microgamétocyte & & Microgamétocyte & & & & $\begin{array}{l}\text { Dans les deux } \\
\text { sexes }\end{array}$ \\
\hline
\end{tabular}


L'évolution des formes asexuées d'une espèce donnée, depuis le trophozoïte jusqu'au schizonte mûr, est relativement aisée à suivre, de nombreux caractères morphologiques du parasite et de sa cellule hôte étant spécifiques et présents pendant tout le développement.

L'identification des gamétocytes est plus difficile. Les critères utilisés ont été, surtout morphologiques, comme par exemple les altérations de la cellule hôte, qui sont comparables chez les formes asexuées et sexuées. Nous sommes conscients du fait que le rattachement des gamétocytes à l'une ou l'autre des espèces présentes, peut être dans certains cas aléatoire et c'est la raison pour laquelle nous avons choisi (cf. plus haut) comme matériel type, un schizonte mûr de chaque espèce.

a -- Plasmodium percygarnhami : chez les trois Lémuriens examinés les deux espèces prédominantes, au cours du premier accès qui suit la splénectomie ou l'inoculation de sporozoïtes, sont $P$. coulangesi et $P$. percygarnhami. Deux types de gamétocytes sont abondants à ce moment.

Les gamétocytes plus petits, entraînant des déformations de l'hématie en feuille de houx, ou en oursin, dépourvus ou présentant peu de granulations azurophiles, ont été rattachés à $P$. percygarnhami parce que les déformations de la cellule hôte sont identiques à celles déterminées par les formes asexuées de cette espèce.

b - Plasmodium bucki : chez le Lémur 3, après traitement par la quinine, une infection apparemment pure à $P$. bucki a été observée. Les gamétocytes sont très différents de ceux décrits pour les autres espèces. Ils sont plus grands, beaucoup plus chromophiles et leur cellule hôte présente des caractères communs avec les formes asexuées de $P$. bucki, en particulier des taches de type Maurer.

\section{Statut taxonomiQue}

Bien que certains stades n'aient pas été observés, les descriptions des premiers auteurs, Buck et coll., 1952 et Huff et Hoogstraal, 1963 sont claires et bien illustrées. Les trois espèces qu'ils ont nommées : Plasmodium girardi et $P$. foleyi chez Lemur fulvus rufus Audebert, 1800, et Plasmodium lemuris chez Lemur fulvus Geoffroy, 1812 sont donc valides.

Une quatrième espèce, $P$. coulangesi Lepers et coll., 1988, vient d'être décrite chez Lemur macaco macaco Linné, 1766.

La présente analyse morphologique et l'examen de la littérature nous amène à admettre 4 autres espèces.

Les huit espèces peuvent être classées en 2 groupes : celles qui déterminent une hypertrophie importante du globule rouge et celles qui évoluent dans un globule rouge de taille peu ou pas modifiée (Tableau I).

A - Espèces ne modifiant pas la taille du globule rouge

a - Plasmodium girardi: Buck et coll., 1952 parasite de Lemur fulvus rufus se caractérise par : la vacuole très nette " paraissant trouer l'hématie » du trophozoïte jeune; le schizonte mûr avec 8 à 10 mérozoïtes et un pigment périphérique ; l'hématie hôte ni hypertrophiée ni décolorée. 
b - Plasmodium coulangesi : Lepers et coll., 1988 parasite de Lemur macaco macaco de la région d'Ambanja se différencie de toutes les autres espèces par la petite taille de ses formes asexuées, le faible nombre (6) de mérozoïtes des schizontes et l'évolution dans un globule rouge non modifié.

c -- Plasmodium percygarnhami n. sp. (= Plasmodium girardi sensu Garnham et Uilenberg, 1975 pro parte, = Plasmodium sp. sensu Lepers et coll., 1989) est un parasite de Lemur macaco macaco de la région d'Ambanja.

Il diffère de Plasmodium girardi Buck et coll., 1952 parasite de Lemur fulvus rufus, par les caractères suivants : - trophozoüles dépourvus d'une vacuole nette "paraissant trouer l'hématie " (cf. Buck et coll., 1952); - schizontes de P. percygarnhami produisant 16 à 20 mérozoïtes et ne remplissant pas la cellule hôte; schizontes de $P$. girardi produisant 8 à 10 mérozoïtes et selon les illustrations de l'article remplissant complètement le globule rouge ; - cellule hôte de $P$. percygarnhami présentant d'importantes déformations caractéristiques en feuille de houx ou en oursin et pouvant être décolorée par les parasites âgés ; cela le différencie de Plasmodium girardi dont le globule rouge hôte est normal ou parfois rétracté.

L'espèce est donc nouvelle et nous la dédions à P. C. C. Garnham.

d - Plasmodium sp. parasite de Lemur fulvus fulvus. Dans leur redescription de $P$. girardi, chez deux hôtes différents, Garnham et Uilenberg ne précisent pas quels sont les stades décrits chez Lemur macaco et chez L. fulvus. L'analyse de leur travail révèle un certain nombre de différences entre leur description et $P$. percygarnhami chez Lemur macaco:- ainsi le nombre de mérozoïtes dans les schizontes mûrs est de 12 à 16 pour eux et le plus souvent de 20 pour nous ; - la forme en " éventail " des schizontes notée par ces auteurs n'a jamais été observée par nous.

Nous pensons donc que le parasite de $L$. m. macaco est différent de celui de Lemur fulvus fulvus et en attendant de pouvoir disposer de matériel provenant de ce dernier ce qui permettrait de décrire l'espèce, nous la désignons sous le nom de Plasmodium sp. (= Plasmodium girardi sensu Garnham et Uilenberg, 1975, pro parte).

B - Espèces augmentant la taille du globule rouge

a - Plasmodium foleyi Buck, Coudurier et Quesnel, 1952 est un parasite très amiboïde, ce qui le différencie de toutes les autres espèces. Il siège dans un globule rouge très hypertrophié et contrairement aux autres espèces du même groupe, l'hématie hôte est décolorée et dépourvue de granulations.

$\mathrm{b}$ - Plasmodium bucki n. sp., parasite de Lemur macaco macaco diffère très nettement de Plasmodium coulangesi, de $P$. percygarnhami et de $P$. girardi par le nombre beaucoup plus élevé de mérozoïtes produits (32), par l'hypertrophie de la cellule hôte et par la présence de taches de type Maurer dans l'hématie parasitée.

Il diffère également des trois espèces entraînant comme lui une hypertrophie de la cellule hôte, $P$. foleyi, $P$. lemuris et $P$. uilenbergi par la présence de taches de type Maurer dans le cytoplasme de la cellule hôte. 
Il se différencie aussi, par d'autres caractères de : 1) Plasmodium foleyi Buck et coll., 1952 : cette espèce est amiboïde, siégeant dans une hématie hôte hypertrophiée et décolorée alors que Plasmodium bucki est peu amiboïde et son hématie hôte est rosée et plus chromophile que les hématies saines qui sont gris clair avec nos colorations ; 2) (?) Plasmodium lemuris: les gamétocytes de cette espèce sont plus grands $(11 \mu \mathrm{m} \times 7 \mu \mathrm{m})$ que ceux de $P$. bucki $(7 \mu \mathrm{m})$; les premiers sont nettement ovalaires, avec un pigment constitué par quelques gros grains, les seconds arrondis, avec un pigment fin ; 3) Plasmodium uilenbergi: les gamétocytes de cette espèce sont plus grands $(11 \mu \mathrm{m})$ que ceux de P. bucki $(7 \mu \mathrm{m})$; contrairement à $P$. bucki, ils présentent un granule chromatinien accessoire (désigné comme centriole par Garnham et Uilenberg, 1975) dans les deux sexes.

L'espèce est donc nouvelle et nous la dédions à G. Buck.

c - Plasmodium uilenbergi n. sp. Il diffère de toutes les autres espèces par : - la présence de grains de Ziemann dans le globule rouge; _ par la présence d'un granule chromatinien accessoire (appelé centriole par Garnham et Uilenberg) dans les gamétocytes des deux sexes : $P$. coulangesi et $P$. percygarnhami ont un granule chromatinien accessoire seulement chez le microgamétocyte et les autres espèces n'en ont pas.

L'espèce est donc nouvelle et nous la dédions à G. Uilenberg : Plasmodium uilenbergi n. sp. (= Plasmodium foleyi sensu Garnham et Uilenberg, 1975 ; = Plasmodium lemuris sensu Uilenberg, 1970).

d-(?) Plasmodium lemuris Huff et Hoogstraal, 1963.

Chez notre L. m. macaco 2 naturellement infecté, de même que chez le Lémur 1, étudié par Lepers et coll., 1989, des gamétocytes de très grande taille, très proches de ceux décrits par Huff et Hoogstraal (1963), ont été observés. N'ayant trouvé aucun schizonte sanguin pouvant être rattaché à cette espèce nous envisageons l'hypothèse selon laquelle l'espèce serait un Haemoproteidae et non un Plasmodiidae. Dans ce cas, le seul schizonte sanguin représenté dans la description originale de P. lemuris (Huff et Hoogstraal, 1963, Fig. \%, p. 253), appartiendrait à une espèce de Plasmodium encore inconnue.

Les caractères morphologiques des gamétocytes de (?) Plasmodium lemuris ne correspondent pas à ceux des Hepatocystis qui sont les seuls Haemoproteidés connus jusqu'à présent chez les Primates; en effet, ils ne remplissent pas le globule rouge, le pigment est peu abondant, irrégulièrement dispersé, chevauchant le noyau. Morphologiquement, il pourrait s'agir du genre Nycteria, mais celui-ci n'a pas encore été signalé chez les Primates.

\section{VICARIANCE DES ESPÈCES}

Les auteurs précédents, et, en particulier Garnham (1966) et Garnham et Uilenberg (1975) ont fait une analyse à tendance synthétique en supposant que les parasites étaient peu spécifiques et que les différences qu'ils observaient entre leurs propres observations et celles de leurs devanciers, étaient dues essentiellement à des variations dépendant des frottis, des colorations et des interprétations. 


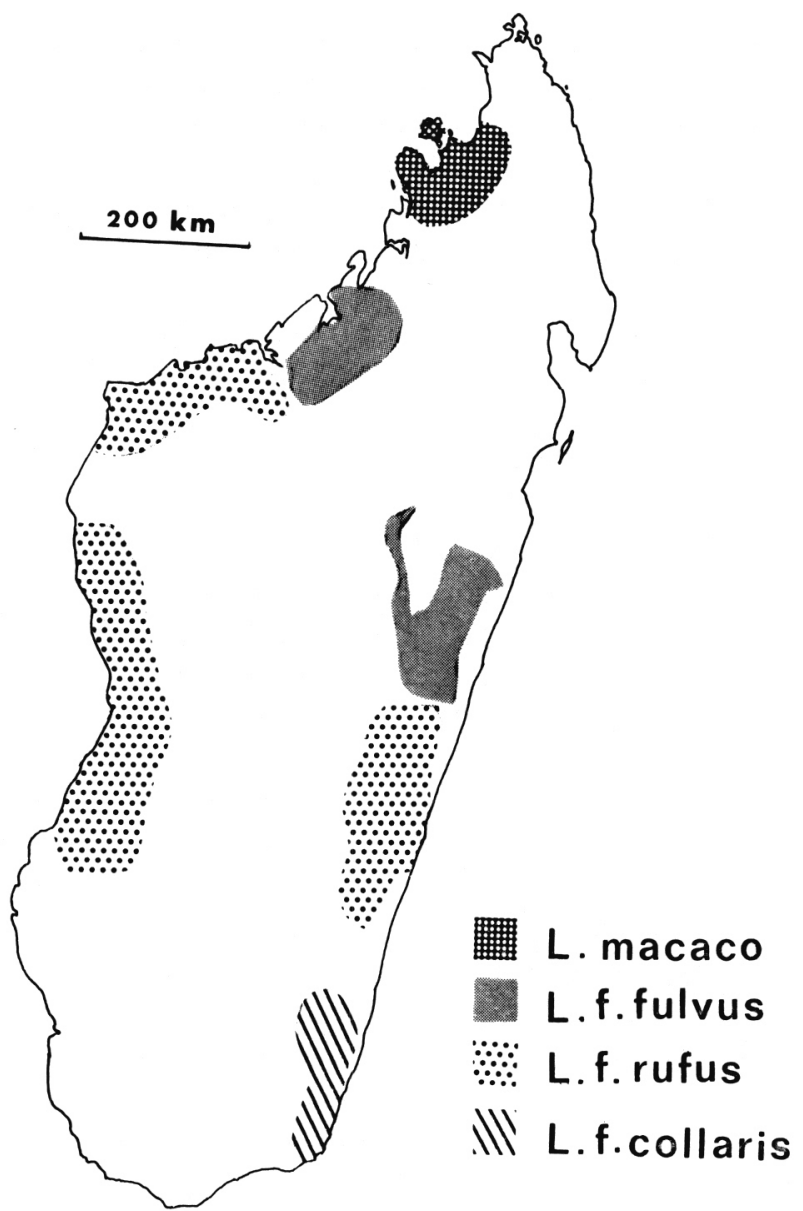

FIG. 1. - Répartition des 4 espèces de Lémuriens paludéens à Madagascar (d'après Petter, Albiniac et Rumpler, 1977).

L'analyse purement morphologique qui précède conduit à une conclusion différente. Nous pensons pouvoir distinguer 8 espèces.

Le cas de Plasmodium lemuris doit être analysé séparément. L'espèce décrite chez L. f. collaris du Sud-Est de l'île est retrouvée sans caractères distinctifs nets chez L. m. macaco du Nord-Ouest. Les gamétocytes ne ressemblent à ceux d'aucun Plasmodium déjà connu. Ce qui a été décrit comme schizonte sanguin par Huff et Hoogstraal ne repose que sur une seule image et pourrait appartenir à un Plasmodium parasite de Lemur fulvus collaris non encore décrit. Nous pensons, donc, sans en avoir la certitude, que Plasmodium lemuris n'est pas un Plasmodium mais plutôt un Nycteria.

Il reste donc 7 espèces de Plasmodium qui ont en commun de nombreux caractères morphologiques. 
Tous ont des gamétocytes du groupe malariae (Landau et coll., 1976), c'està-dire : — ne remplissent pas le globule rouge; — présentent une limite nette entre noyau et cytoplasme du microgamétocyte ; - possèdent un pigment grossier, réparti de façon non uniforme et chevauchant le noyau.

Deux des espèces, $P$. coulangesi et $P$. percygarnhami ont été étudiées en microscopie électronique (Boulard et coll., sous presse). Elles présentent des caractères beaucoup plus primitifs que les espèces connues chez d'autres Mammifères, en particulier, des mitochondries avec des crêtes ampullaires, identiques à celles qui sont observées chez les parasites d'Oiseaux et de Reptiles.

Les sept espèces, bien que proches les unes des autres se distinguent aisément. Elles se répartissent en deux groupes selon que le globule rouge hôte est de taille normale ou hypertrophié.

Le Tableau I rassemble la liste des hôtes et les principaux caractères différentiels entre les espèces de Plasmodium.

On constate que chaque espèce ou sous-espèce de Lémurien est parasitée par un couple formé d'une espèce déterminant une hypertrophie du globule rouge et une espèce ne la provoquant pas. Lemur m. macaco a en outre, une troisième espèce dont l'homologue n'est pas connu chez les autres Lémuriens.

L'analyse purement morphologique conduit à la notion que le paludisme des Lémuriens est étonnamment proche de celui qui est connu chez les Rongeurs africains. Il y a chez chaque Rongeur au moins un couple vicariant caractéristique (Landau, 1966). Ces phénomènes de spéciation ont été difficilement mis en évidence chez les Plasmodium de Rongeurs; les analyses enzymatiques (Beale et coll., 1978) ont été précieuses pour confirmer le statut spécifique et subspécifique de chaque taxon.

Dans le cas des Lémuriens malgaches, la répartition des hòtes selon la localisation géographique (Fig. 1) est bien définie (cf. Petter et coll., 1977). Il en résulte une spéciation particulièrement nette des Plasmodium.

Remerciements. - Nous exprimons toute notre reconnaissance à Jean-Jacques Petter et Roland Albignac pour leur aide et leurs conseils ; leur connaissance profonde des Lémuriens nous a particulièrement aidés tout au long de ce travail. Nous remercions Maryvonne Leclerc-Cassan et François Hugues du Zoo de Vincennes, Anne Michelap et Laurent Daumas du Service Chirurgical de l'Hôpital de la Pitié-Salpêtrière qui ont effectué la splénectomie et la biopsie hépatique des Lémuriens hébergés au Muséum ; grâce à eux le couple de Lemur macaco a été maintenu en bonne santé et bénéficie d'une retraite paisible au Parc Zoologique.

\section{RÉFÉRENCES}

Buck G., Coudurier J., Quesnel J.-J. : Sur deux nouveaux Plasmodium observés chez un Lémurien de Madagascar splénectomisé. Arch. Inst. Pasteur Algér., 1952, 30, 240-243.

Beale G. H., Carter R., Walliker D. : Genetics in Rodent Malaria, Academic Press, London, New York, San Francisco, 1978, 406 p.

Boulard Y., Landau I., Rabetafika L., Baccam D., Lepers J.-P., Coulanges P. : Études ultrastructurales sur les Plasmodium de Lémuriens malgaches. European Journal of Protistology, 1989 (sous presse). 
Garnham P. C. C. : Malaria parasites and other Haemosporidia. Blackwell Scientific Publications, Oxford, 1966, $1114 \mathrm{p}$.

Garnham P. C. C., Vilenberg G. : Malaria parasites of Lemurs. Ann. Parasitol. Hum. Comp., $1975,50,409-418$.

Huff C. G., Hoogstraal H. : Plasmodium lemuris n. sp. from Lemur collaris E. Geoffroy. J. infect. Dis., 1963, 112, 233-236.

LANDAU I. : Comments on sporozoite induced infections in Rodent Hosts. Military Medecine, $1966,131,919-922$

Landau I., Miltgen F., Chabaud A. G. : Les différents types de gamétocytes chez les Hémosporidies de Mammifères. Corrélations avec la morphologie des schizontes tissulaires. Hypothèses sur l'évolution du groupe. Ann. Parasitol. Hum. Comp., 1976, 51, 175-187.

Lepers J. P., Rabetafika L., Landau I., Peters W. : Une nouvelle espèce Plasmodiale chez un Lémurien : Plasmodium coulangesi n. sp. : Ann. Parasitol. Hum. Comp., 1989, 64, 163-170.

Petter J.-J., Albignac R., Rumpler Y. : Mammifères lémuriens (Primates prosimiens) in Faune de Madagascar, ORSTOM, CNRS, 1977, 44, 513 p.

Rabetafika L., Landau I., Baccam D., Lepers J. P., Cycle biologique expérimental des plasmodies de Lemur macaco macaco. Ann. Parasitol. Hum. Comp., 1989 (sous presse).

Uilenberg G. : Quelques Protozoaires parasites de Mammifères sauvages à Madagascar. Ann. Parasitol. Hum. Comp., 1970, 45, 183-194. 